\title{
Dominant Rate Process of Silicon Surface Etching by Hy- drogen Chloride Gas
}

Hitoshi Habuka, Takahiro Suzuki, Sunao Yamamoto, Akio Nakamura, Takashi TAkeuchi and Masahiko AiHara

Department of Chemical Engineering Science, Yokohama National University, 79-5 Tokiwadai, Hodogaya, Yokohama, Kanagawa 240-8501, Japan

Silicon surface etching and its dominant rate process are studied using hydrogen chloride gas in a wide concentration range of $1-100 \%$ in ambient hydrogen at atmospheric pressure in a temperature range of $1023-1423 \mathrm{~K}$, linked with the numerical calculation accounting for the transport phenomena and the surface chemical reaction in the entire reactor. The etch rate, the gaseous products and the surface morphology are experimentally evaluated. The dominant rate equation accounting for the first-order successive reactions at silicon surface by hydrogen chloride gas is shown to be valid. The activation energy of the dominant surface process is evaluated to be $1.5 \times 10^{5} \mathrm{~J} \mathrm{~mol}^{-1}$. The silicon deposition by the gaseous by-product, trichlorosilane, is shown to have a negligible influence on the silicon etch rate.

Etch rate, silicon, hydrogen chloride, dominant rate process 


\section{Introduction}

Hydrogen chloride gas is a widely used etchant in various processes[1-4] for manufacturing semiconductor silicon crystal and highly integrated microelectronics devices. For developing and advancing future processes using hydrogen chloride gas, a practical simulation technology for the hydrogen chloride processes is expected. This demand requires the rate equation and its rate constants, which can reproduce and predict the etch rate and its behavior by the numerical calculation accounting for the entire transport phenomena in the reactor. This information by the numerical calculation will help designing a chemical process using the various chemical reaction on the silicon surface. Therefore, a rate theory on the chemical reaction between the silicon surface and the hydrogen chloride gas should be studied, for the future advance in the silicon technology.

The silicon etch process by hydrogen chloride gas has been reported, in a number of studies[5-22]. Although there are many experimental data of the silicon surface etch rate and behavior, they should be recognized as a group of fragments. Most of them have been proposed a second order chemical reaction[11-15, 17-21] to produce $\mathrm{SiCl}_{2}$, based on the nonlinear behavior of the etch rate in the small hydrogen chloride gas concentration region and based on the equilibrium theory. However, the dominant etch product was detected to be trichlorosilane and tetrachlorosilane[22], not $\mathrm{SiCl}_{2}$; the etch rate greater than 2 or $3 \mu \mathrm{mmin}^{-1}$ showed linear relationship with the partial pressure of hydrogen chloride gas[18]. Furthermore, the surface reaction rate constant has not been evaluated using the numerical calculation taking the transport phenomena in the reactor into account. Therefore, in order to build a model for silicon etch process by hydrogen chloride gas, the entire behavior of the etch rate should be newly obtained and evaluated, using a fixed reactor having simple gas flow and heat which are suitable for performing the numerical calculation taking the transport phenomena and the surface chemical reaction rate into account. This information will be valuable for the high rate silicon etching including the reactor 
cleaning[1-3].

First, we evaluate the etch rate, the surface morphology, the gaseous products, by means of an experiment, in this study. Next, the dominant rate equation and the overall rate constant of silicon etching by hydrogen chloride gas are evaluated, for the first time, using a numerical calculation taking the transport equations and the surface reaction on the silicon surface into account.

\section{Experimental details}

In order to study the etching of the silicon surface by hydrogen chloride gas, a horizontal cold-wall reactor as shown in Fig. 1 was used. This reactor consists of a gas supply system, a quartz chamber and an infrared lamp-heating module. The quartz chamber has a rectangular cross section of $0.04 \mathrm{~m}$ width and $0.01 \mathrm{~m}$ height. Its very small height is designed to effectively suppress natural convection. A $0.03 \mathrm{~m} \times$ $0.05 \mathrm{~m}$ semiconductor single crystal silicon substrate, cut from the $200 \mathrm{~mm}$-diameter silicon wafer, is held horizontally on the bottom surface of the quartz chamber. The outside walls of the quartz chamber are cooled by supplying cold air to achieve a cold-wall environment. The gas supply system has the function of introducing hydrogen chloride gas along with hydrogen gas for etching the silicon substrate in the quartz chamber. A trace amount of water vapor with a dew point less than $213 \mathrm{~K}$, included in the high purity hydrogen gas (Sumitomo Seika Kogyo Co., Ltd., Tokyo), was removed using a liquid nitrogen $(77 \mathrm{~K})$ trap as shown in Fig. 1. High purity hydrogen chloride gas (Sumitomo Seika Kogyo Co., Ltd., Tokyo) vaporized from the liquid gas container was used without further purification.

The entire process performed in this study, shown in Fig. 2, mainly consisted of three steps:

(a) removing the native oxide and the organic contamination on the silicon substrate surface by baking for $10 \mathrm{~min}$ at $1373 \mathrm{~K}$ in ambient hydrogen,

(b) adjusting the silicon substrate temperature used for the next step by changing 
the electric power supplied to the infrared lamps, and

(c) etching the silicon substrate surface using hydrogen chloride gas mixed with hydrogen gas.

Since a very wide concentration range of hydrogen chloride gas is suitable in order to experimentally estimate its reaction order and the changes in the dominant chemical products and process, the concentration of the hydrogen chloride gas at the inlet of the reactor was $1-100 \%$. The total flow rate of the gas mixture was $0.002 \mathrm{~m}^{3} \mathrm{~min}^{-1}$ at atmospheric pressure throughout this study. Although the hydrogen pressure at each hydrogen chloride gas concentration is the parameter to be taken into account, it was only adjusted in order to keep atmospheric pressure in the reactor. This is mainly for avoiding explosion due to gas leak from this reactor system. The silicon substrate temperature was in the range of $1023-1423 \mathrm{~K}$. These conditions include the industrially-applicable condition for cleaning the silicon substrate, the susceptor and the chamber of the chemical vapor deposition reactor.

The silicon etch rate using the hydrogen chloride gas was evaluated by measuring the difference in the weight of the silicon substrate before and after the etching. The weight difference was $10-70 \%$ of the silicon substrate. The etch rate obtained in this study is the average value over the silicon substrate surface.

In order to measure the chemical species in the gas phase without inducing any unfavorable influence[23] on the gas flow and the chemical reaction at and above the silicon substrate, part of the exhaust gas from the reactor was fed to the quadrupole mass (QMS) analyzer (Microvision, Spectra International LLC), as shown in Fig. 1, similar to our previous study[7]. For the measurement of the mass spectra to clearly detect the existence of various gaseous species and to minimize their fragmentation due to the ionization, the ionization energy and current were set at $18 \mathrm{eV}$ and $0.25 \mathrm{~mA}$, respectively. This measurement system and condition enable to detect $\mathrm{SiCl}_{2}$ and $\mathrm{SiCl}$ when they are produced in the cold wall reactor, because of the minimized fragmentation in the QMS and the suppression of the gas phase chemical reaction due to the low temperature in the cold wall thermal environment[7]. 


\section{Numerical Calculation}

The geometry of the horizontal cold wall reactor, shown in Fig. 1, was taken into account for a series of calculation for evaluating the chemical process in this study. In order to evaluate the silicon etch rate in a steady state in non-uniformly distributed temperature and gas flow fields, two- and three-dimensional equations of the mass, momentum, energy, species transport and surface chemical reaction were solved simultaneously, the same as the transport and epitaxy model $[5,6]$ for silicon epitaxial film growth.

The gas velocity and pressure at the inlet were $0.08 \mathrm{~ms}^{-1}$ and $1.0133 \times 10^{5} \mathrm{~Pa}$, respectively. The temperature change by the heat of reaction is assumed to be negligible. Since accurate measurement of the temperature of the transparent quartz glass walls is extremely difficult, their temperatures were estimated empirically and assumed to vary linearly with the silicon substrate temperature in the range between $590 \mathrm{~K}$ and $750 \mathrm{~K}[5,6]$. The temperature of the other reactor walls was regarded as $300 \mathrm{~K}$. The temperature of the chamber wall between the inlet and the silicon substrate and that between the outlet and the silicon substrate were treated as a heat conducting wall with the thermal conductivity of quartz glass.

The etching of a silicon substrate surface is assumed to follow the overall reaction in equation (1).

$$
\mathrm{Si}+3 \mathrm{HCl} \rightarrow \mathrm{SiHCl}_{3} \uparrow+\mathrm{H}_{2} \uparrow .
$$

Mass changes due to the chemical reaction of equation (1) on the silicon substrate surface are considered in the boundary conditions at the silicon substrate surface. Taking the experimental results in this study into account, the overall reaction shown in equation (1) is assumed to be a first-order reaction.

The concentration of each species at the surface is governed by a balance between the consumption by the chemical reaction and the diffusion fluxes driven by the concentration and temperature gradients. The boundary condition for conservation 
of species $i$ on the substrate surface is

$$
\nu_{i} M_{i} k[\mathrm{HCl}]=-\rho D_{i} \frac{\partial \omega_{i}}{\partial z}-D_{i}^{\mathrm{T}} \frac{\partial \ln T}{\partial z} .
$$

where $k$ is the overall rate constant for the reaction of equation (1), and the values of the molar stoichiometry coefficient, $\nu_{i}$, are 1 for $i=\mathrm{SiHCl}_{3}$ and $\mathrm{H}_{2}$, and -3 for $i=\mathrm{HCl} . \quad M_{i}$ is the molecular weight of species $i\left(\mathrm{~kg} \mathrm{~mol}^{-1}\right) . \quad[\mathrm{HCl}]$ is the mole concentration of hydrogen chloride gas at the substrate surface $\left(\mathrm{molm}^{-3}\right) . \quad \rho$ is the density of the mixture of hydrogen chloride, hydrogen and trichlorosilane gas $\left(\mathrm{kgm}^{-3}\right) . \quad D_{i}$ is the diffusion coefficient of species $i\left(\mathrm{~m}^{2} \mathrm{~s}^{-1}\right), D_{i}^{\mathrm{T}}$ is the thermal diffusion coefficient of species $i\left(\mathrm{~kg} \mathrm{~m}^{-1} \mathrm{~s}^{-1}\right) . \omega_{i}$ is the mass fraction of species $i(-)$. $T$ is the temperature in the reactor $(\mathrm{K}) . z$ is the coordinate in the vertical direction.

The etched amount of silicon per unit time and area of the surface,

$$
R_{\mathrm{Si}}=M_{\mathrm{Si}} k[\mathrm{HCl}]
$$

gives the etch rate of silicon surface as

$$
\text { Etch rate }=6 \times 10^{7} R_{\mathrm{Si}} / \rho_{\mathrm{Si}} \quad\left(\mu \mathrm{mmin}^{-1}\right)
$$

where $\rho_{\mathrm{Si}}$ is the density of solid silicon, and the factor $6 \times 10^{7}$ is used for the unit conversion of $\mathrm{ms}^{-1}$ to $\mu \mathrm{m} \mathrm{min}^{-1}$.

To solve the transport equations described above, the calculation domain was divided into a non-uniform grid with 4,800 and 96,000 grid points for the two- and three-dimensional calculation, respectively. Preliminary calculations using different numbers of grid points showed that the grid points used in this study were sufficient to obtain accurate calculation results. In this study, the discretized equations were coupled and solved using the SIMPLE algorithm[24] on a computational fluid dynamics software package, Fluent version 5.5 (Fluent, Inc., Lebanon, NH, USA). Additionally, the calculations are performed using the code[6] based on the SIMPLER algorithm[24].

The gas properties of hydrogen, trichlorosilane, hydrogen chloride and their gas mixture, such as viscosity, thermal conductivity and heat capacity, were taken from 
the literature[25, 26]. The properties of the mixed gas were estimated theoretically [27]. The diffusion coefficients of trichlorosilane and hydrogen chloride in hydrogen are estimated with the method described in the literature[28].

In this study, the overall rate constant, $k$, in equation (3) is obtained so that the calculated etch rates agree with those measured at various temperatures.

\section{Results and Discussion}

In this section, the etching process is discussed based on various results, such as the silicon etch rate behavior, the surface morphology and the gaseous products. After deriving the rate equation taking the dominant chemical process on the silicon substrate surface into account, the overall rate constant is evaluated using the numerical calculation.

\subsection{Silicon etch rate}

The silicon substrate surface is etched by the hydrogen chloride gas in ambient hydrogen at atmospheric pressure using the cold wall reactor shown in Fig. 1, in the temperature range of 1023-1423 K. Circles, squares, triangles and diamonds in Fig. 3 show the silicon etch rate measured in this study.

The etch rate at each temperature in this study increased with the hydrogen chloride concentration set at the inlet of the reactor. The largest etch rate obtained in this study was $80 \mu \mathrm{mmin}^{-1}$ at the silicon substrate temperature of $1423 \mathrm{~K}$ at the hydrogen chloride concentration of $100 \%$. The notable behavior of the silicon etch rate shown in this figure is its almost linear increase at each temperature even for the conditions from very large (100\%) to very small (near 1\%) hydrogen chloride gas concentration. Therefore, the results obtained in this study indicate that the overall chemical reaction order of the hydrogen chloride gas can be assumed to be one as written in equation (2), since the almost linear increase of the silicon etch rate over the wide hydrogen chloride gas concentration range is observed in Fig. 3. This reaction order will be verified in the later part of this study. 


\subsection{Surface morphology}

This section briefly describes the relationship between the silicon etch rate and the change in the surface morphology of the silicon substrate during the etching, since the surface morphology provides the fundamental information on the increase and the decrease in the surface area influencing the surface chemical reaction rate. Since the changes in the surface morphology are observed to be intense by visual inspection, it is observed using an optical digital microscope (VH-8000, Keyence Co., Ltd., Tokyo), as shown in Fig. 4. This figure shows the surface morphology etched at (a) the silicon substrate temperature of $1423 \mathrm{~K}$ for $15 \mathrm{~min}$ with the hydrogen chloride concentration of $5 \%$, and at (b) $1273 \mathrm{~K}$ for $75 \mathrm{~min}$ with $1 \%$.

As reported in our previous study[3], the morphology of the silicon substrate surface becomes rough with the increasing etch period. Similarly, the silicon substrate surface in this study was observed to become rough due to the etching at $1423 \mathrm{~K}$ for 15 min with the hydrogen chloride concentration of $5 \%$ as shown in Fig. 4 (a). Fig. 4 (b) also shows pits on the silicon substrate surface etched at $1273 \mathrm{~K}$ for $75 \mathrm{~min}$ with the hydrogen chloride concentration of $1 \%$. As shown in Fig. 4, the surface morphology at $1273 \mathrm{~K}$ is rougher than that at the higher temperature of $1423 \mathrm{~K}$. A smooth surface was obtained at higher substrate temperatures rather than the lower temperatures, in this study. This trend agrees with the report by Lang and Stavish[13] and Putte et al.[19]. If the silicon surface roughening increased the reactive surface area, the silicon etch depth shows an exponential-like increase with the increasing etch period, due to an increase in the surface area. However, the linear increase in the silicon etch depth with the etch period was experimentally observed at the hydrogen chloride concentration causing the surface roughening. Therefore, the influence of the surface roughening on the silicon etch rate is considered to be negligible. 


\subsection{Gaseous chemical products}

In order to study the chemical reactions at the silicon substrate surface and in the gas phase, the gaseous chemical species produced in the reactor are evaluated using the QMS, as shown in Fig. 1. Fig. 5 shows the chemical species measured under the condition using the hydrogen chloride concentration of $50 \%$ and the silicon substrate temperature of $1373 \mathrm{~K}$. The partial pressure in this figure is normalized using that for the mass of 36 which shows the greatest partial pressure in this measurement.

As shown in Fig. 5, five groups clearly existed at masses near 2, 36, 98, 133 and 168. The partial pressures at masses of $1-2$ and 35-38 are assigned to hydrogen gas and hydrogen chloride gas supplied from the inlet of the reactor. The great partial pressures at the masses of 133-140 and 168-176 are assigned to the $\mathrm{SiCl}_{3}^{+}$group and $\mathrm{SiCl}_{4}^{+}$group, respectively. Taking the influence of the fragmentation in the QMS analyzer into account, the major chemical species existing in the exhaust gas are considered to be trichlorosilane $\left(\mathrm{SiHCl}_{3}\right)$ and tetrachlorosilane $\left(\mathrm{SiCl}_{4}\right)$. In spite of the difference of the thermal condition, such as cold wall and hot wall, this result coincides with those by Nishizawa and Nihira[22].

The partial pressures at the masses of $98-102$, the $\mathrm{SiCl}_{2}^{+}$group, were much lower than those of the $\mathrm{SiCl}_{3}^{+}$group and $\mathrm{SiCl}_{4}^{+}$group. Because of the very small partial pressure of the $\mathrm{SiCl}_{2}^{+}$group and the negligibly small partial pressure of the $\mathrm{SiCl}^{+}$ group having the masses of $63-66, \mathrm{SiH}_{2} \mathrm{Cl}_{2}, \mathrm{SiCl}_{2}$ and $\mathrm{SiCl}$ are not considered to be the dominant product in the silicon etching process. Because the major gaseous products detected by the QMS were the same for every measurement using the wide ranges of the temperature and the hydrogen chloride concentration, the dominant surface chemical process of the silicon etching by hydrogen chloride gas at atmospheric pressure is concluded to unchange in the temperature range used in this study. The excess amount of hydrogen chloride gas supplied is considered to proceed the surface chemical process to form the final product of trichlorosilane, in contrast to the atomic layer etching by chlorine[29]. 


\subsection{Chemical process}

Next, the dominant chemical process to etch the silicon substrate surface by hydrogen chloride gas is described based on the information obtained in this study and the existing studies[11-15, 29]. In order to produce trichlorosilane and tetrachlorosilane, the successive reactions can be assumed as shown in Fig. 6 and as follows:

$$
\begin{gathered}
\mathrm{Si}+\mathrm{HCl} \rightarrow{ }^{*} \mathrm{SiCl}+\frac{1}{2} \mathrm{H}_{2} \uparrow . \\
{ }^{*} \mathrm{SiCl}+\mathrm{HCl} \rightarrow{ }^{*} \mathrm{SiCl}_{2}+\frac{1}{2} \mathrm{H}_{2} \uparrow . \\
{ }^{*} \mathrm{SiCl}_{2}+\mathrm{HCl} \rightarrow \mathrm{SiHCl}_{3} \uparrow .
\end{gathered}
$$

Here, the symbol * indicates the chemisorbed state on the silicon substrate surface. The hydrogen chloride produces trichlorosilane, which cannot continue to stay on the silicon substrate surface because of its low boiling point of $305 \mathrm{~K}$ and very large vapor pressure.

Since there is a very large amount of hydrogen chloride gas in the gas phase above the silicon substrate surface, trichlorosilane is considered to react with hydrogen chloride to produce tetrachlorosilane.

$$
\mathrm{SiHCl}_{3}+\mathrm{HCl} \rightarrow \mathrm{SiCl}_{4}+\frac{1}{2} \mathrm{H}_{2}
$$

Since the silicon epitaxial growth can occur in the trichlorosilane-hydrogen system, the following two reactions may simultaneously occur on the silicon substrate surface, as a reverse process of the silicon etching:[5, 6, 30-32]

$$
\begin{gathered}
\mathrm{SiHCl}_{3} \rightarrow{ }^{*} \mathrm{SiCl}_{2}+\mathrm{HCl} \uparrow \\
{ }^{*} \mathrm{SiCl}_{2}+\mathrm{H}_{2} \rightarrow \mathrm{Si}+2 \mathrm{HCl} \uparrow
\end{gathered}
$$

Here, equation (8) occurs only in the gas phase. Additionally, the rate of equations (9) and (10) are considered to be suppressed due to the excess amount of hydrogen chloride gas in the reactor in this study. Therefore, equations (5)-(7) are considered to be dominant during the silicon etching by the hydrogen chloride gas. 


\subsection{Dominant rate equation and overall rate constant}

In this section, the dominant rate equation of the silicon etch rate by hydrogen chloride gas is obtained and evaluated, following the rate theory[33]. The chemical reactions in equations (1), (5), (6) and (7) and the assumption for the surface morphology influence are concluded to be valuable, when the rate equation derived from these equations can reproduce the experimentally obtained etch rate in Fig. 3.

Assuming the successive surface chemical reactions in equations (5)-(7), and taking the fraction of surface reaction sites occupied by ${ }^{*} \mathrm{SiCl}$ and ${ }^{*} \mathrm{SiCl}_{2}$ into account, the silicon etch rate is described as equation (3) where the overall rate constant of silicon etching, $k$, is expressed in equation (11).

$$
k=\frac{1}{1 / k_{1}+1 / k_{2}+1 / k_{3}},
$$

where $k_{1}, k_{2}$ and $k_{3}$ are the rate constants $\left(\mathrm{m} \mathrm{s}^{-1}\right)$ of equations (5), (6) and (7), respectively. Equation (11) predicts that the overall rate constant does not depend on the surface concentrations of hydrogen chloride gas, hydrogen gas and trichlorosilane gas. Next, the value of the overall rate constant in equation (11) is evaluated so that the measured silicon etch rate in Fig.3 is reproduced by the numerical calculations.

The calculation in this study showed that the gas flow in the reactor was parallel to the heated silicon substrate surface and that natural convection was very well suppressed, following the design concept of this reactor.

The obtained values of the overall rate constant are depicted as the Arrhenius plot in Fig. 7. From this figure, the overall rate constant is shown to follow the Arrhenius law, as follows:

$$
k=6 \times 10^{4} \exp \left(\frac{-1.5 \times 10^{5}}{R T}\right) \mathrm{ms}^{-1}
$$

The activation energy obtained in this study is $1.5 \times 10^{5} \mathrm{Jmol}^{-1}$. This value is consistently larger than the apparent activation energy of $1.7 \times 10^{4} \mathrm{~J} \mathrm{~mol}^{-1}$ reported by Putte et al[19], because the activation energy by Putte et al. included the influence 
of not only the surface reaction, but also the entire transport phenomena in the reactor.

The solid lines in Fig. 3 are the calculation using the $k$ value of equation (12). Since the calculation is recognized to reproduce the etch rate changing with the silicon substrate temperature and the hydrogen chloride concentration in this study, the $k$ value obtained in this study is considered to be applicable to describe the behavior of the silicon etch rate by hydrogen chloride gas, and is concluded to be independent of the surface concentrations of hydrogen chloride, hydrogen and trichlorosilane gases, as predicted in equation (11). Therefore, the rate equations (3) and (11), based on the rate process shown in Fig. 6, are concluded to be valid. Simultaneously, the silicon deposition due to the gaseous product of trichlorosilane is considered to have a negligible influence on the silicon etch rate in this study.

The very slight non-linearity of the measured etch rate in Fig. 3 is considered to be due to the transport phenomena in the gas phase, similar to the silicon epitaxial growth[6]. Especially, the trichlorosilane gas and tetrachlorosilane gas produced at and around the silicon surface are considered to be very slowly transported away, because they have the very smaller diffusion coefficient than the rest of gases in the reactor.

The influence of ambient gas on the silicon etch rate has been reported to exist [19]. However, since silicon etch rate is very large at the hydrogen chloride gas concentration of $100 \%$, silicon etching is concluded to be possible without hydrogen gas, in contrast to the silicon epitaxial growth in a chlorosilane-hydrogen system[21]. In order to further clarify the dominant chemical process of silicon surface etching, the behavior of the silicon etch rate using various ambient gases is considered to be necessary.

\section{Summary}

The etch rate of the silicon substrate surface using hydrogen chloride gas and its rate process are studied using ambient hydrogen at atmospheric pressure in a 
temperature range of $1023-1423 \mathrm{~K}$ and a hydrogen chloride concentration range of $1-100 \%$. The rate theory of silicon surface etching using hydrogen chloride gas is studied by means of calculations using transport equations and the surface chemical reaction rate. The dominant surface chemical process is assumed to consist of the first-order successive reaction to produce trichlorosilane. The activation energy of the overall rate constant is evaluated to be $1.5 \times 10^{5} \mathrm{~J} \mathrm{~mol}^{-1}$. Since the rate equation obtained in this study can reproduce the silicon etch rate, the rate equation and the rate process are concluded to be valid. Simultaneously, the silicon deposition by the gaseous by-product, trichlorosilane, is shown to have a negligible influence on the silicon etch rate.

\section{Acknowledgment}

The authors thank the Kawasaki Steel 21st Century Foundation for supporting this study. They thank Mr. N. Okumura of Keyence Co., Ltd. for taking the photographs using the optical digital microscope. The technical support of the software, Fluent, by Y. Miura of Fluent Asia Pacific, Inc. is noted here and greatly appreciated. The authors thank H. Koda, D. Saito, M. Kawaoka, S. Fukaya and A. Sawada of Yokohama National University for giving us fruitful advices. 


\section{References}

[1] H. Hasegawa, T. Yamaoka, M. Hamano, Y. Ishihara and T. Satoh, Proceedings of the ninth International Symposium on Semiconductor Manufacturing., Tokyo Japan, Sep. 26-28 2000, p. 379.

[2] H. Habuka, T. Otsuka and M. Katayama, J. Cryst. Growth, 186 (1998) 104.

[3] H. Habuka, H. Tsunoda and T. Otsuka, J. Electrochem. Soc., 145 (1998) 4264.

[4] J. Nishizawa and H. Nihira, J. Cryst. Growth, 45 (1978) 82.

[5] H. Habuka, T. Nagoya, M. Mayusumi, M. Katayama, M. Shimada and K. Okuyama, J. Cryst. Growth, 169 (1996) 61.

[6] H. Habuka, M. Katayama, M. Shimada and K. Okuyama, J. Cryst. Growth, 182 (1997) 352 .

[7] H. Habuka, Y. Aoyama, S. Akiyama, T. Otsuka, W. F. Qu, M. Shimada and K. Okuyama, J. Cryst. Growth, 207 (1999) 77.

[8] H. Habuka, S. Fukaya, A. Sawada, T. Takeuchi and M. Aihara, Jpn. J. Appl. Phys., 41 (2002) 5692.

[9] P. Ho, A. Balakrishna, J. M. Chacin, A. Thilderkvist, B. Haas and P. B. Comita, Electrochem. Soc. Proc., 98-23 (1998) 117.

[10] S. Kommu, G. M. Wilson and B. Khomami, J. Electrochem. Soc., 147 (2000) 1538 .

[11] V. S. Ban and S. L. Gilbert, J. Cryst. Growth, 31 (1975) 284.

[12] J. P. Duchemin, M. Bonnet and G. Beuchet, J. Vac. Sci. Technol., 16 (1979) 1126.

[13] G. A. Lang and T. Stavish, RCA Rev., 24 (1963) 488.

[14] C. Dominguez, G. Pastor and E. Dominguez, J. Electrochem. Soc., 134 (1987) 199.

[15] C. Dominguez, G. Pastor and E. Dominguez, J. Electrochem. Soc., 134 (1987) 202.

[16] T. Kataoka, Y. Sato and T. Ohmine, Electrochem. Soc. Proc., 97-25 (1997) 
1319.

[17] I. H. Oh and C. G. Takoudis, J. Appl. Phys., 69 (1991) 8336.

[18] J. Bloem, J. Cryst. Growth, 18 (1973) 70.

[19] P. van der Putte, L. J. Giling and J. Bloem, J. Cryst. Growth, 41 (1977) 133.

[20] J. Bloem and W. A. P. Claasen, J. CrystalGrowth, 49 (1980) 435.

[21] J. Korec, J. Cryst. Growth, 61 (1983) 32.

[22] J. Nishizawa and H. Nihira, J. Cryst. Growth, 45 (1978) 45.

[23] T. O. Sedgwick, J. E. Smith, Jr., R. Ghez and M. E. Cowher, J. Cryst. Growth, $31(1975) 264$.

[24] S. V. Patankar, Numerical Heat Transfer and Fluid Flow, McGraw-Hill New York, 1980.

[25] Kagaku Binran 3rd ed., Iwanami, Tokyo, 1984, p. II-39, 71 and 239.

[26] P. Pollard and J. Newman, J. Electrochem. Soc., 127 (1980) 744.

[27] C. R. Wilke, J. Chem. Phys., 18 (1950) 517.

[28] R. C. Reid, J. M. Prausnitz and B. E. Poling, The Properties of Gases and Liquids 4th ed., McGraw-Hill, New York, 1987.

[29] T. Shirao, K. Shudo, Y. Tanaka, T. Ishikawa, and M. Tanaka, Jpn. J. Appl. Phys., 42 (2003) L386.

[30] Y. Ohshita and N. Hosoi, J. J. Cryst. Growth, 131 (1993) 495.

[31] E. Sirtl, L. P. Hunt and D. H. Sawyer, J. Electrochem. Soc., 121 (1974) 919.

[32] P. Kongetira, G. W. Neudeck and C. G. Takoudis, J. Vac. Sci. Technol., B15 (1997) 1902.

[33] G. M. Barrow, Physical Chemistry 6th ed., McGraw-Hill, New York, 1996. 


\section{Figurecaptions}

Fig. 1. Horizontal cold wall chemical vapor deposition reactor used in this study for etching silicon substrate surface. This reactor consists of a silicon substrate, a quartz chamber, halogen lamps and gas supply system of hydrogen gas and hydrogen chloride gas. A quadrupole mass spectrometer is used for analyzing the gaseous chemical species in the exhaust gas.

Fig. 2. Process for cleaning and etching of the silicon substrate surface in this study.

Fig. 3. Etch rate of silicon surface at the silicon substrate temperatures of 1023 $1423 \mathrm{~K}$ by hydrogen chloride gas in ambient hydrogen at atmospheric pressure. Circles, squares, triangles and diamonds are the measurement. Solid lines are the calculation.

Fig. 4. Silicon substrate surface etched by hydrogen chloride gas in ambient hydrogen at atmospheric pressure using the optical digital microscope. The temperature and the hydrogen chloride concentration to etch the silicon substrate surface is (a) $1373 \mathrm{~K}$ with $5 \%$ for $15 \mathrm{~min}$ and (b) $1273 \mathrm{~K}$ with $1 \%$ for $75 \mathrm{~min}$, respectively.

Fig. 5. Quadrupole mass spectra of the exhaust gas during the etching of silicon substrate surface at the hydrogen chloride concentration of $50 \%$ at the silicon substrate temperature of $1373 \mathrm{~K}$.

Fig. 6. Chemical process of etching and epitaxy at the silicon surface and in the gas phase for a hydrogen chloride-trichlorosilane-hydrogen system.

Fig. 7. Arrhenius plot of the rate constant of silicon surface etching by hydrogen chloride gas. 


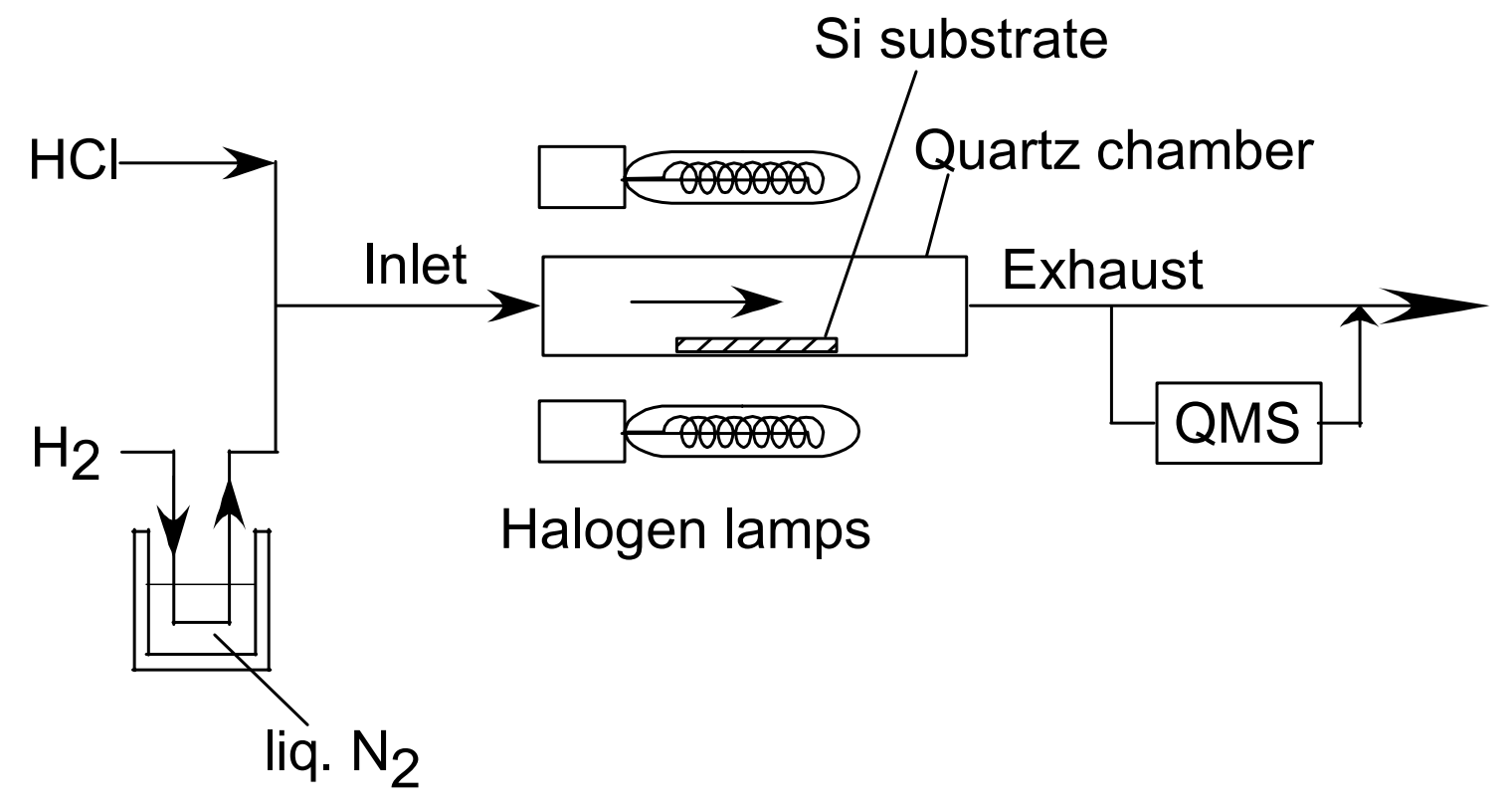

Habuka et al.,

Fig. 1 


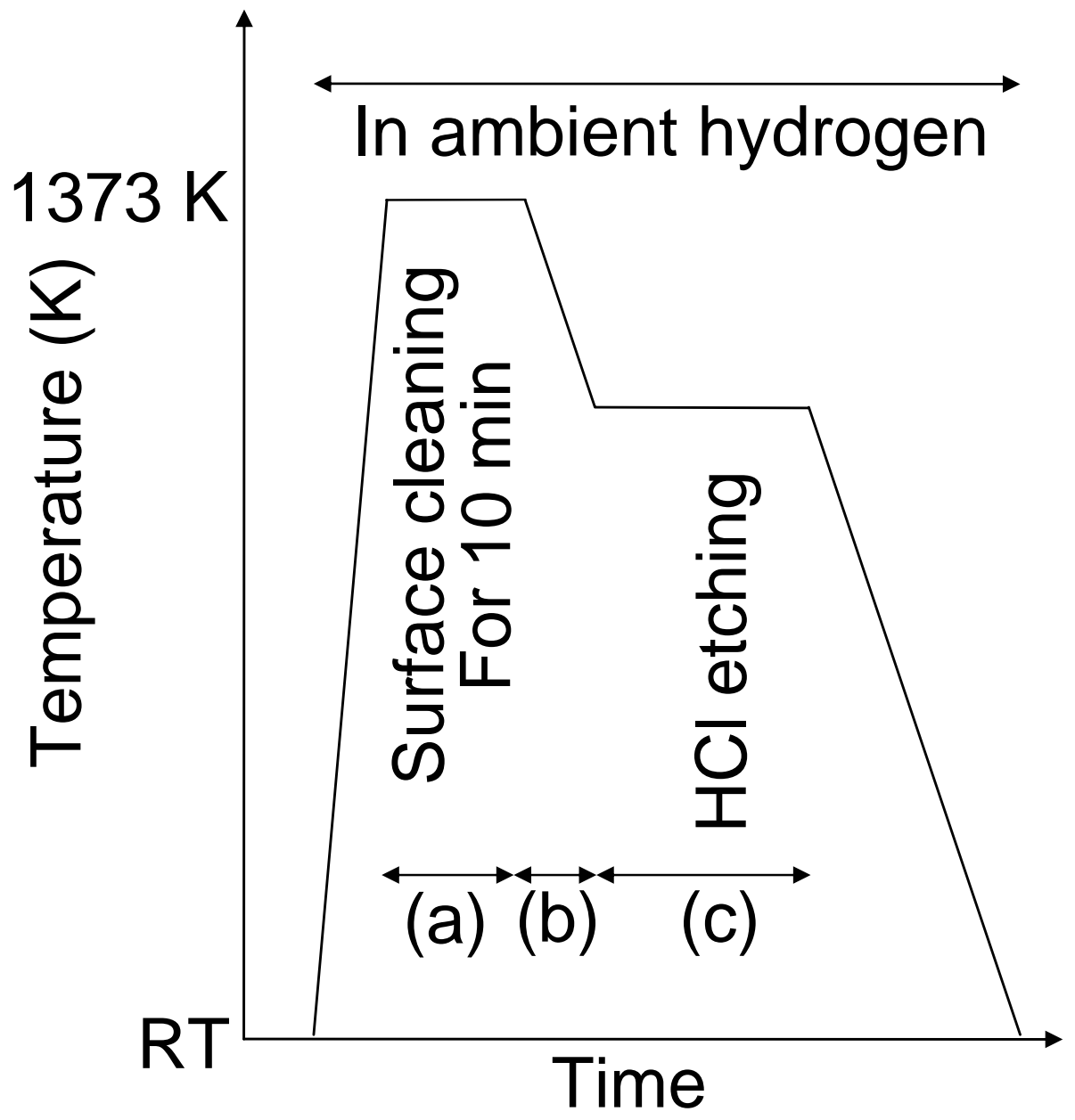

Habuka et al.,

Fig. 2 


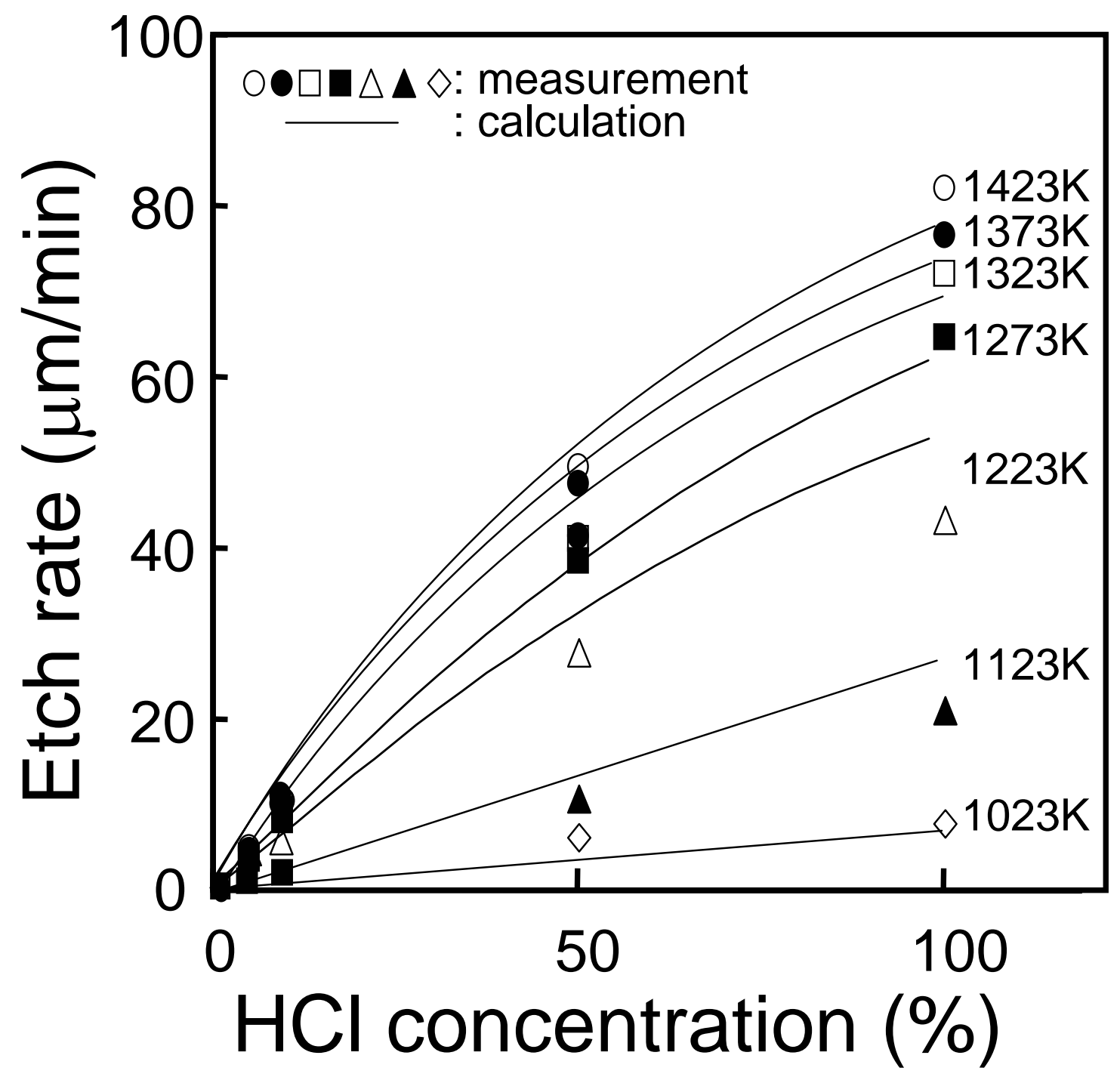

Habuka et al.,

Fig. 3 


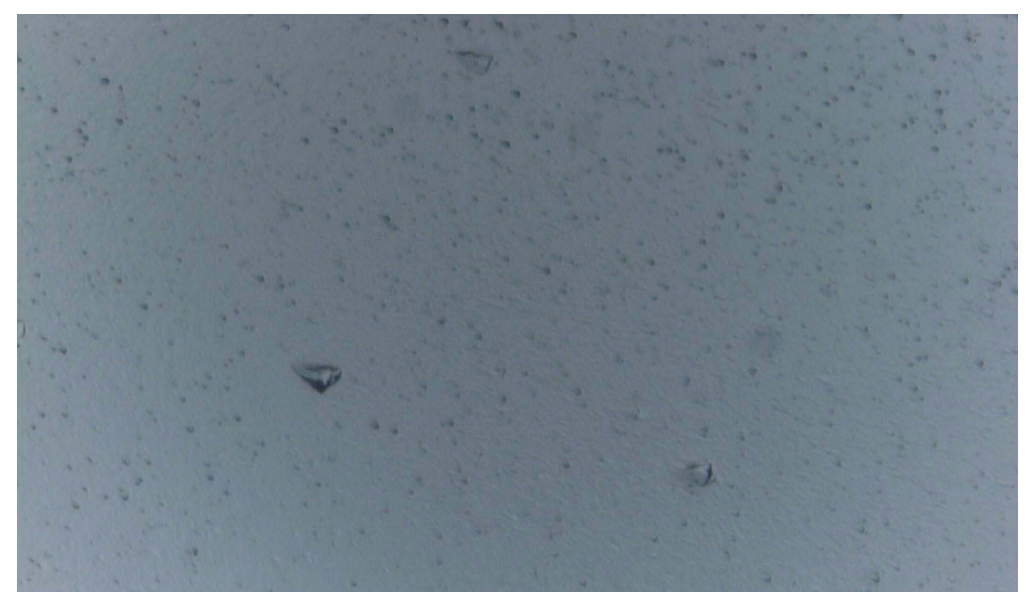

\section{(a) $1423 \mathrm{~K}, 5 \%$}

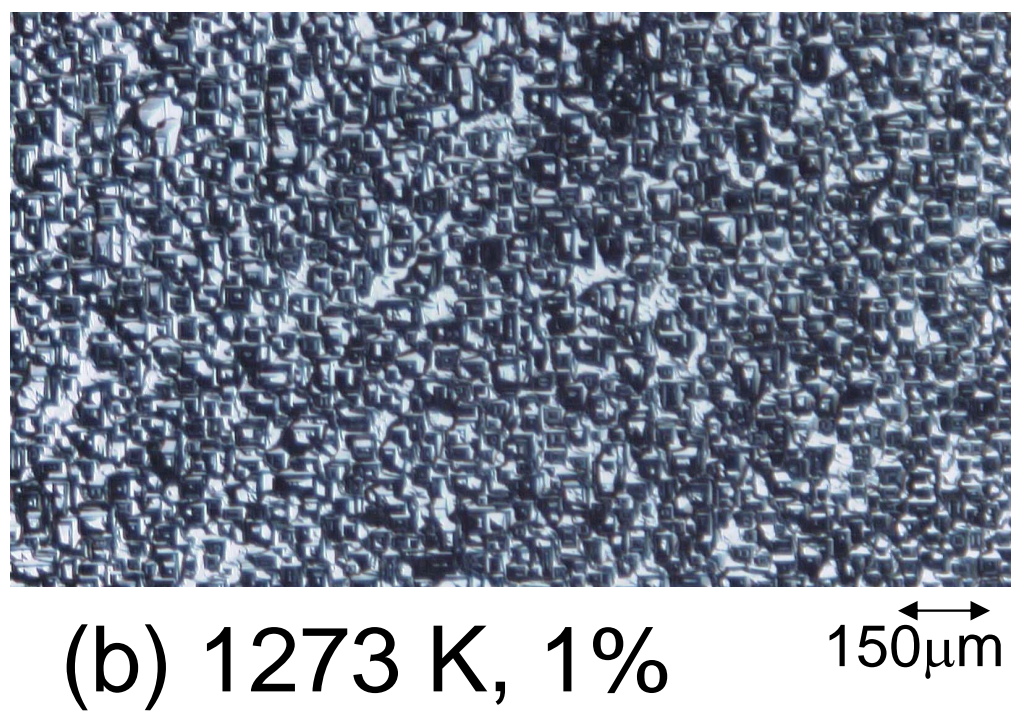

Habuka et al.,

Fig. 4 


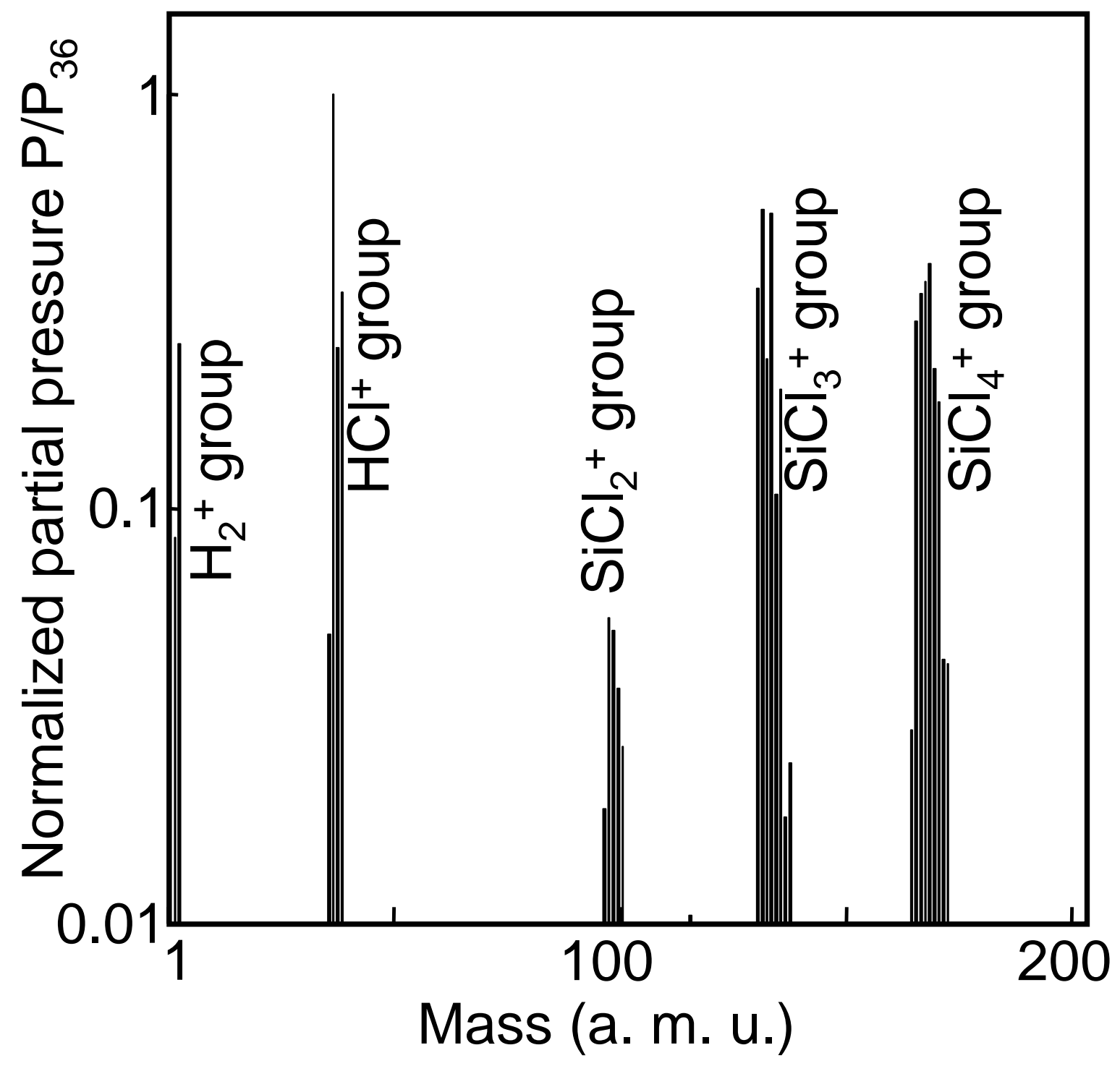

Habuka et al.,

Fig. 5 


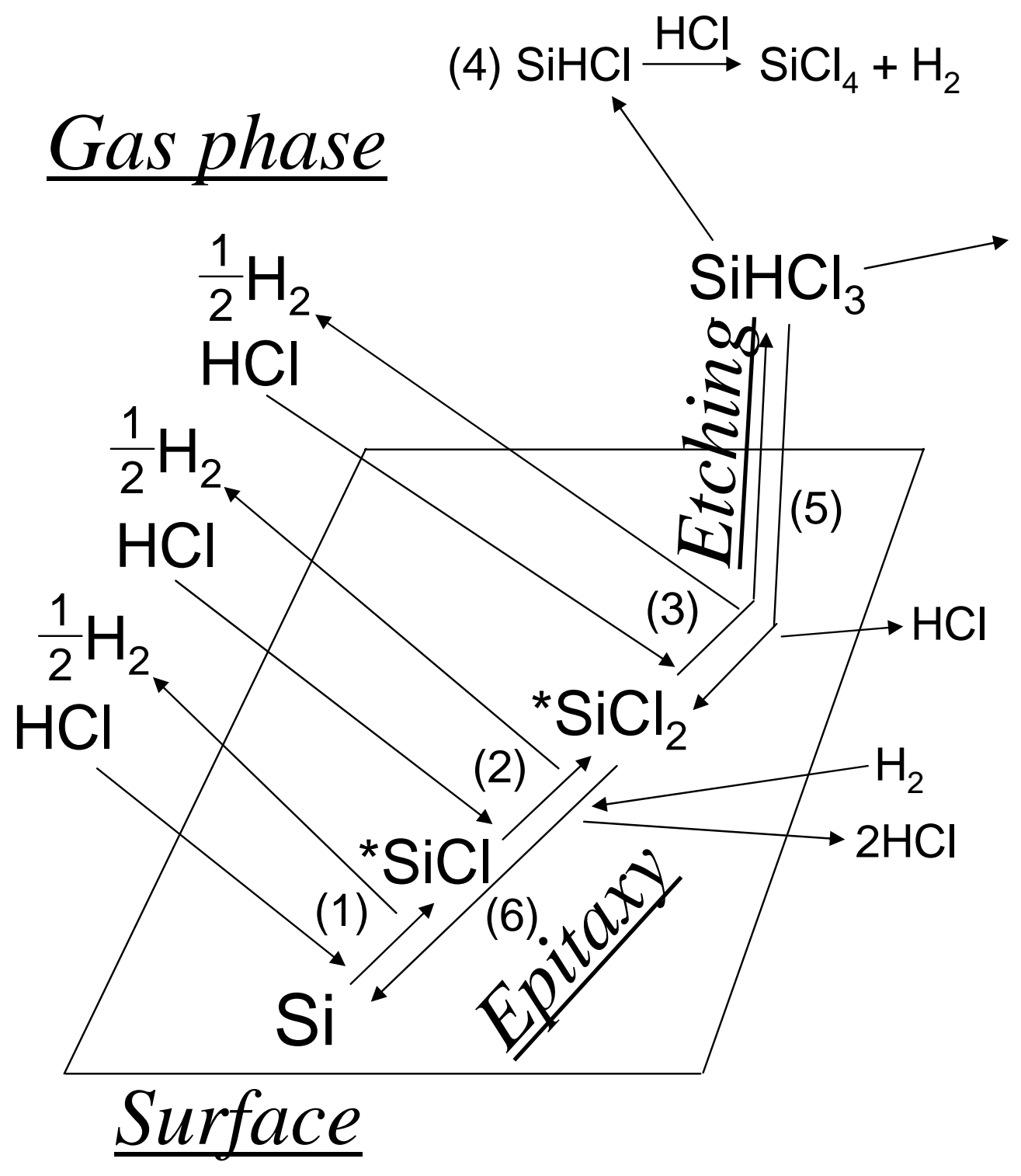

Habuka et al.,

Fig. 6 


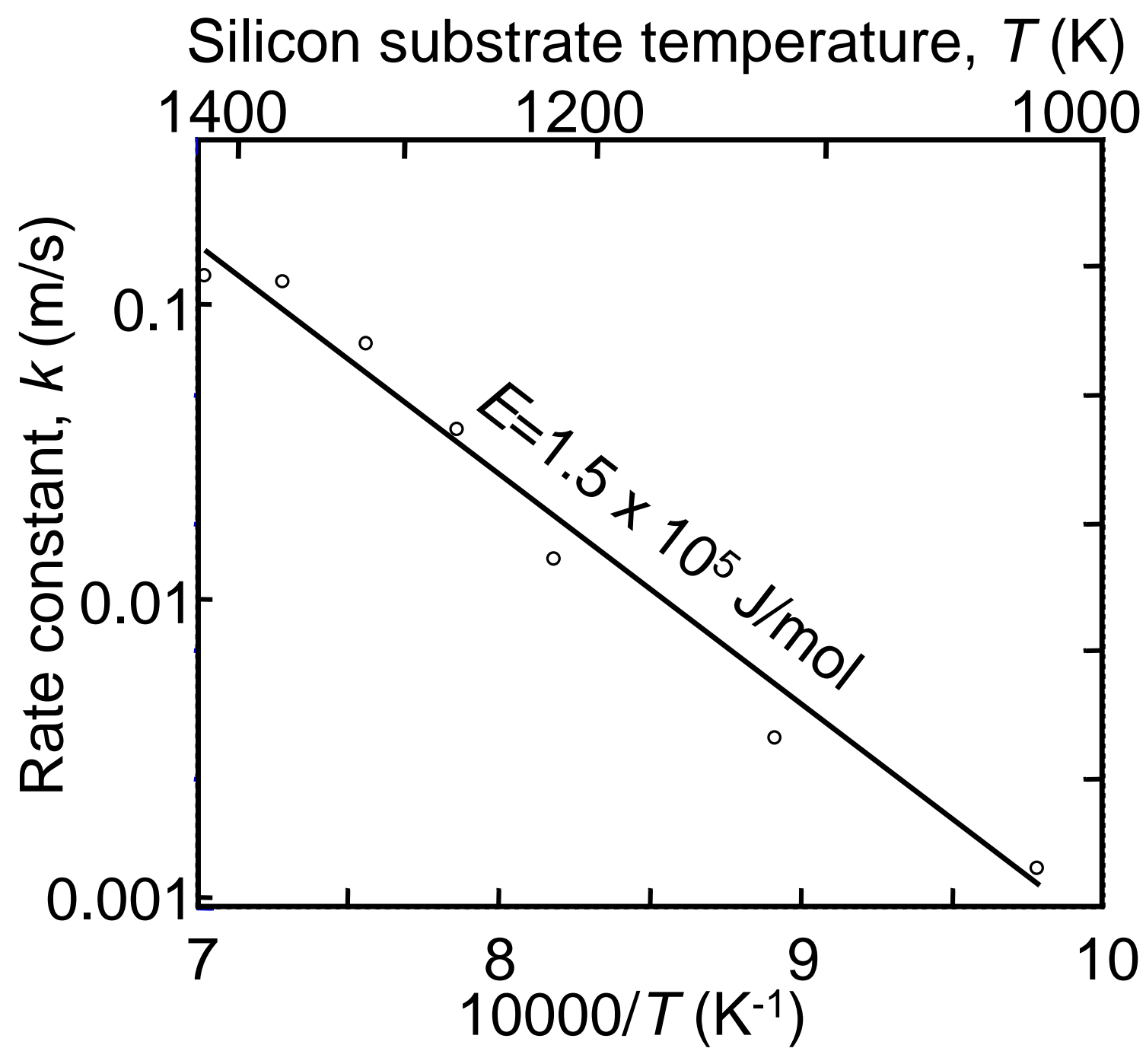

Habuka et al.,

Fig. 7 